\title{
Correcting tensile test results of ECAE-deformed aluminium
}

\author{
Stijn Poortmans, ${ }^{\mathrm{a},}$ Boubakar Diouf, ${ }^{\mathrm{b}}$ Anne-Marie Habraken ${ }^{\mathrm{b}}$ and Bert Verlinden ${ }^{\mathrm{a}}$ \\ ${ }^{\mathrm{a}}$ Katholieke Universiteit Leuven, Metaalkunde en Toegepaste Materiaalkunde, Kasteelpark Arenberg 44, B3001 Heverlee, Belgium \\ ${ }^{\mathrm{b}}$ University of Liège, Département d'Architecture, Géologie, Environnement and Constructions, Chemin des Chevreuils 1, 4000 Liège, \\ Belgium
}

\begin{abstract}
Performing tensile tests on ECAE material reveals a long post-uniform elongation. In order to calculate correct true stress-true strain diagrams, three different approaches are used: measurements of the actual sample geometry, a neck evolution model proposed by Segal [V.M. Segal, S Ferrasse, F. Alford, Mater. Sci. Eng. A442 (2006) 321-326] and an inverse modelling method by finite element (FE) simulations.
\end{abstract}

Keywords: SPD; Strain hardening; Tension test

Until recently it was believed that grain size refinement towards ultrafine-grained material leads to a loss of strain hardening capacity. However, when closely observing the shape change during tensile deformation at room temperature, it can be seen how a very small homogeneous deformation occurs, followed by a long postuniform elongation [2]. Calculating a stress-strain curve from a load-displacement curve assuming homogeneous deformation obviously leads to an erroneous shape of the flow curve (see Fig. 1). A correction method has recently been proposed by Segal et al. [1] by looking at the evolution of the sample boundaries. This leads to a modified set of equations for the calculation of stress, strain and strain rate in the sample neck. In the present paper, experimental verifications by measuring the actual sample cross-section are performed. They provide an improved tensile true stress-true strain curve for AA1050 aluminium after equal channel angular extrusion (ECAE) processing. The inverse finite element FE method provides both the material stress-strain curve and the actual sample cross-section. The computed results depend on the material model assumed. Finally, experimental results are compared with both FE and Segal's approach, allowing their validation.

The material used in this study is commercial-purity aluminium (type AA1050). It was delivered hot rolled and fully recrystallized with an initial grain size of 100-300 $\mu \mathrm{m}$. All ECAE samples were machined with the extrusion direction (ED) parallel to the former rolling direction (RD). The samples had a diameter of $12 \mathrm{~mm}$ and a length of $60 \mathrm{~mm}$. ECAE was performed at room temperature following route $B_{\mathrm{C}}$ [3] for 8 passes, lubricating with $\mathrm{MoS}_{2}$. The ECAE die had an intersection angle $\Phi=90^{\circ}$, without rounding $\left(\Psi=0^{\circ}\right)$. The final grain size was about $1 \mu \mathrm{m}$, with more than $70 \%$ high angle grain boundaries (when analysed by EBSD as described in Ref. [4]).

The tensile samples were machined such that only the homogeneous zone of the ECAE sample would be subjected to deformation. Stainless steel custom-made extension parts with screw fixation were used for mounting. All the tests were performed at room temperature on an Instron 4505 machine with $100 \mathrm{kN}$ load cell. The initial strain rates were $2.5 \times 10^{-5}$ and $8.3 \times 10^{-2} \mathrm{~s}^{-1}$ respectively, for constant crosshead speeds of $2.5 \times 10^{-7}$ and $8.3 \times 10^{-4} \mathrm{~m} \mathrm{~s}^{-1}$ during the test. For the FE simulations only the high crosshead speed was retained.

To determine the initial yield point, the slope of the stress-strain curve during elastic deformation was used to calculate back $0.2 \%$ plastic deformation while accounting for slip and elastic deformation in the extension parts and screw fixation of the sample. In order to measure sample anisotropy and the inhomo-geneity of deformation due to necking, Laser interfero-metry was used [5]. This approach resulted in a points cloud with an average density of 75 points $/ \mathrm{mm}^{-2}$, with an individual spatial resolution of $0.05 \mathrm{~mm}$. The cross-sectional sample shape was observed to become elliptical during tensile deformation and the corresponding mathematical description was determined by the use of a least mean squares approximation [6].

Compared with hot-rolled aluminium, ECAE-pro-cessed aluminium has a different behaviour in tensile deformation [4], showing a long post-uniform elongation. Therefore the actual true stress-true strain relationship has to be determined without assuming homogeneous deformation and thus accounting for the diffuse necking. 
Figure 1: True stress-true strain curves, computed with different assumptions, (a) Geometrical methods: assumed homogeneous deformation, Segal correction, interrupted tensile tests; (b) Segal correction, FE method optimized elasto-plastic material law when using Segal as initial input, FE method optimized elasto-visco-plastic material law of Norton-Hoff type.
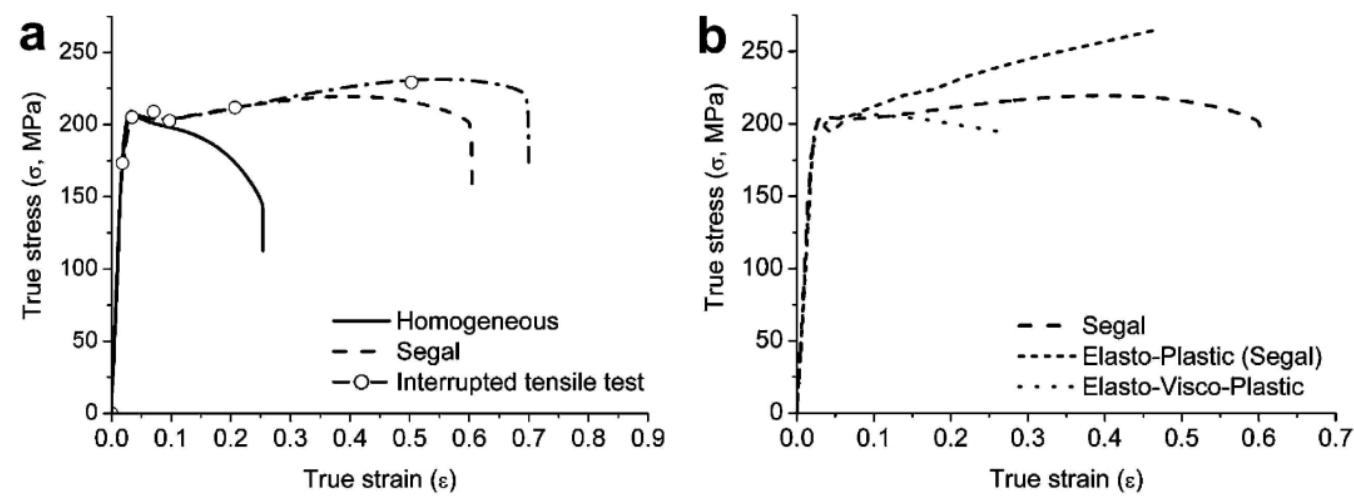

In the present paper the following approach was used. The Considère criterion telling that a transition between homogeneous and non-homogeneous deformation can happen after the maximum of the force-displacement curve was applied. For the homogeneous deformation the conventional formulae are used to convert the loaddisplacement data into a true stress-true strain curve. After the force maximum, the formulae proposed by Segal et al. [1] are used. The result of these calculations can be seen in Figure 1. It is obvious that the elastic part and the uniform deformation part of both curves overlap. However, when looking at the non-uniform deformation a clear difference between the conventional and the adapted approach is visible. The latter shows hardening, after a short transition region with softening. An ultimate tensile strength of about $220 \mathrm{MPa}$ is reached, and the sample has a fracture strain of 0.6

An alternative method to correct the true stress-true strain curve is by performing interrupted tensile tests. Here the sample is deformed to a certain plastic strain, unloaded and measured in the smallest cross-section.

These results are shown in Table 1. In contrast to conventional Al-alloys, the ECAE samples show an elliptical cross-section. " $A$ " and " $B$ " correspond to the main axes of the ellipse [2]. $D_{\text {eq }}$ is calculated as the diameter of a circle with the same cross-section area as the ellipse. From these values and the measured load, the real true stress and true strain are calculated, assuming no local necking has occurred. These discrete points, as well as an interpolated stress-strain curve, are plotted in Figure 1. Also here a softening region followed by hardening is observed. The results agree well with what is simulated by Segal's method, although at higher strains some deviation between both results is observed.

When using the Segal approach it is possible to obtain the actual sample cross-section and actual (active) neck length. The (local) strain is used to calculate the local cross-section area and from this the average circular sample radius can be obtained. The result of this approach is shown in Figure 2 for an assumed homogeneous plastic strain of $16 \%$. In order to calculate the actual active deforming length $l_{\mathrm{i}}$, the following assumption is made. Since the actual deforming length behaves as a new tensile sample for each small increment, the conservation of volume can be assumed, leading to the following equation for $l_{\mathrm{i}}$ :

$$
l_{\mathrm{i}}=\frac{\mathrm{d} l}{\exp (\varepsilon)-1}
$$

The equation is only valid during diffuse necking, not for local necking. For homogeneous deformation the initial value of the active length $\left(l_{\mathrm{i}}\right)$ corresponds to the initial gauge length. 
Figure 2: The tensile sample profile at 16\% total strain as calculated from the Segal method, measured crosssections and the measured profile by Laser-Interferometry.

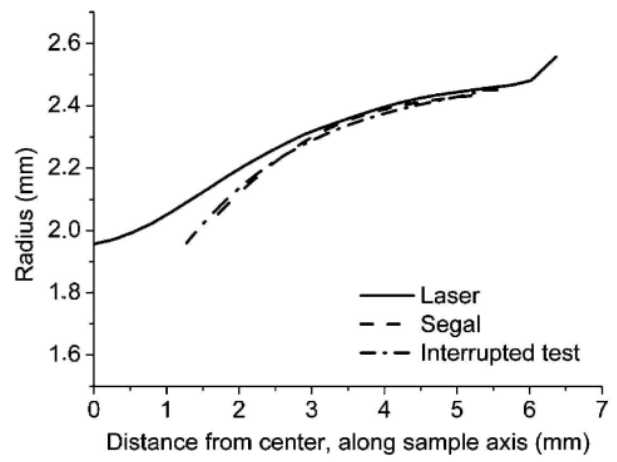

Table 1: Actual smallest cross-section values from interrupted tensile tests

\begin{tabular}{|c|c|c|c|c|c|c|c|c|c|}
\hline \multirow[t]{2}{*}{ Value $\left(\varepsilon_{\text {plastic }}\right)$} & \multirow[t]{2}{*}{$A(\mathbf{m m})$} & \multirow[t]{2}{*}{$B(\mathrm{~mm})$} & \multicolumn{3}{|c|}{$D_{\mathrm{eq}}(\mathrm{mm})$} & \multirow[t]{2}{*}{$\varepsilon_{\text {homog }}$} & \multirow{2}{*}{$\begin{array}{l}\sigma_{\text {homog }} \\
(\mathrm{MPa})\end{array}$} & \multirow[t]{2}{*}{$\varepsilon_{\text {interrupt }}$} & \multirow{2}{*}{$\begin{array}{c}\sigma_{\text {interrupt }} \\
\text { (MPa) }\end{array}$} \\
\hline & & & Exp. & EP & EVP & & & & \\
\hline Yield & 5.00 & 5.00 & 5.00 & 5.00 & 5.00 & $1.8 \%$ & 173.3 & $1.8 \%$ & 173.3 \\
\hline Neck & 4.91 & 4.91 & 4.91 & 4.87 & 4.89 & $3.4 \%$ & 205.2 & $3.4 \%$ & 205.2 \\
\hline $2 \%$ & 4.90 & 4.85 & 4.87 & 4.84 & 4.87 & $4.1 \%$ & 202.8 & $5.01 \%$ & 209.0 \\
\hline $4 \%$ & 4.84 & 4.78 & 4.81 & 4.69 & 4.77 & $7.0 \%$ & 197.1 & $7.8 \%$ & 202.6 \\
\hline $8 \%$ & 4.66 & 4.45 & 4.55 & 4.47 & 4.59 & $11.2 \%$ & 192.7 & $18.7 \%$ & 211.9 \\
\hline $16 \%$ & 4.09 & 3.77 & 3.93 & 3.83 & 4.03 & $20.5 \%$ & 170.2 & $48.3 \%$ & 229.3 \\
\hline
\end{tabular}

Comments: $D_{\mathrm{eq}}=$ the equivalent circular diameter, calculated from the elliptical cross-section shape with major axis $A$ and minor axis $B$; $\varepsilon=$ $\varepsilon_{\text {elastic }}+\varepsilon_{\text {plastic }}$; subscript 'homogeneous': assuming homogeneous deformation: subscript 'interrupt': local values from interrupted tests; Yield: $0.2 \%$ plastic strain: Neck: calculated according to the Considère criterion.

An experimental verification of the diameter profile was obtained by laser interferometry (LI) on a tensile sample. Calculating a $D_{\text {eq }}$ as described above, the results from LI are compared with the simulated values. These results are shown in Figure 2. At the initial deformation the calculated profiles correspond well to the LI measured profile. Some deviation from the LI profile is caused by the fact that the (diffuse) necking did not take place in the exact centre of the specimen and because the sample geometry has a small rounding radius at each shoulder.

In order to get an even better approximation of the actual sample geometry and the true stress and true strain, an FE simulation can be used [6,7]. The principle is based on inverse modelling. Starting with a material law defined by some initial parameters, the tensile test is simulated by FE simulation using the geometry shown in Figure 3a. The used axisymmetric mesh is shown in Figure 3b. A normalized square root of the difference between the numerical and experimental curves defines a cost function. By modification of the material parameters, an optimizer tool finds the set of values minimizing the cost function. In this article, the FE Lagamine software [8] coupled with a Levenberg-Marquardt optimizer algorithm [9] is used.

When the experimental target is reduced to the force-displacement curve, the stress-strain curve (elasto-plastic (Segal)) is above the interrupted tensile test curve and the Segal stress-strain curve (see Fig. 1b). Moreover, with this material model, the simulation predicts a tensile sample profile quite far from the measured one (see Fig. 4b and Table 1). If the experimental radius evolution of the central sample cross-section is added to the forcedisplacement curve in the cost function of the optimizer, one can observe a very strong effect of the initial values of the parameters of the material law provided in the optimization process. Whether a perfect plastic curve or hardening curve is taken as the starting curve, they both converge towards a final hardening curve, providing a compromise predicting both the force-displacement curve and the profile curve but with a high quadratic error (0.901 and 0.871, respectively). If one uses the Segal curve as the initial value, the presence of initial softening followed by hardening allows the optimizer to escape from local minima in the cost function and yields towards a better result predicting the experimental curves (see Fig. 4: FE Segal (optimized)). The quadratic error is 
reduced to 0.487. FE simulations prove that the shape of the Segal curve is the only one that allows for the recovery of both the experimental measurements of the force and the profile.

Further investigation using the exact strain rate history during the test and an elasto-visco plastic (EVP) model of the Norton type, Eq. (2), shows that in this case the optimizer is no more sensitive to the initial parameters set and recover without problem the "force-displacement" target curve and a "radius-time".

$$
\sigma=P_{2} \varepsilon^{P_{4}} \mathrm{e}^{-P_{1} \varepsilon} \sqrt{3}(\sqrt{3} \dot{\varepsilon})^{P_{3}}
$$

The set of Norton parameters obtained in this study is $P_{1}=0.647, P_{2}=90 \mathrm{MPa}, P_{3}=0.033$ and $P_{4}=0.0403$.

Figure 3: (a) Sample geometry, with testing diameter $5 \mathrm{~mm}$ and testing length $10 \mathrm{~mm}$ and (b) FE axisymmetric mesh using a four-node, assumed-strain element.
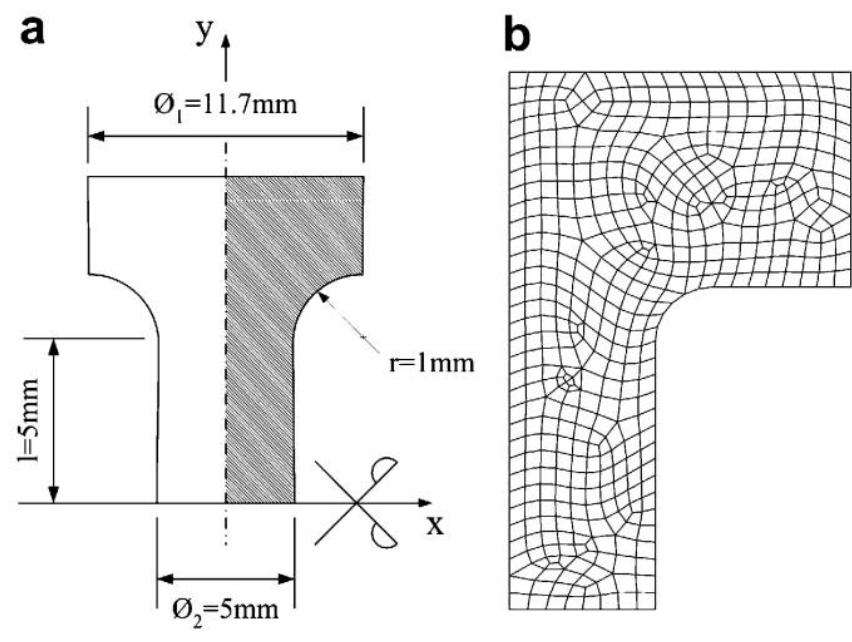

Figure 4: (a) Force-displacement curves as obtained experimentally and resulting from FE simulations using both non-optimized and optimized Segal material laws and the EVP Norton-Hoff law; and (b) the obtained tensile sample profiles as measured by laser and as obtained from the FE results.
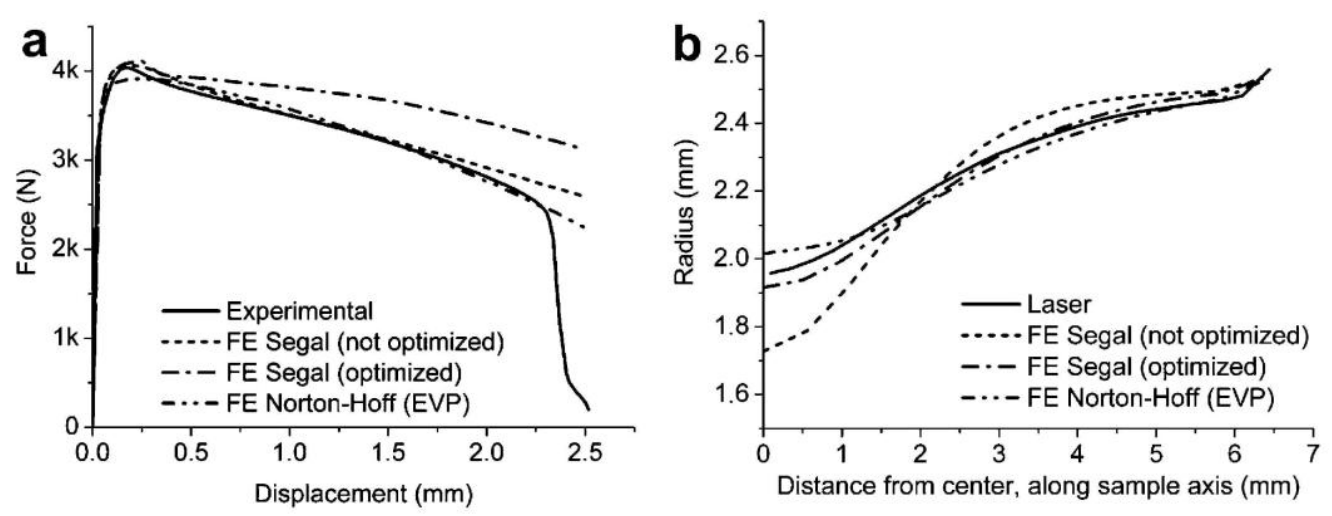

With this model, the error is 0.505 which is close to the best result provided by the previous elasto-plastic (EP) model. The resulting graphs are shown in Figure 4 and the profile values are summarized in Table 1.

When comparing the non-FE-calculated stress-strain curves, it can be concluded that Segal gives a good approximation for the real material behaviour. At larger strains the Segal method underestimates the locally 
acting stresses (Fig. la). This is also visible on the tensile sample profile (Fig. 3), where only minor differences between the Segal and the interpolated approach are visible. The largest deviation between the LI-measured profile and the calculated profile is in the medium strain range. There, the calculations underestimate the strain hardening rate and give more severe necking at lower displacement values.

A successful optimization on both the force-displacement and tensile sample profiles is not possible at the same time when using an elasto-plastic material law in FE. However, starting from the Segal material law provides quite a good result, although this is not perfect. For the elasto-plastic material law, when using the inverse FE method, the initial starting curve must already be close to the global minimum. Other initial values stop at local minima, still higher than the minimum provided when starting from the Segal material law.

It can be seen from Figure $\mathrm{lb}$ that a local minimum must be present in the material law after the elastic behaviour. This local minimum, linked to some material softening, must be related to the change in strain path from simple shear in ECAE towards unidirectional tensile strain in the tensile test. A possible explanation for this behaviour can be the well-known cross effect [10]. When calculating the Schmitt factor, it can be seen that it transforms from -0.55 during ECAE route $B_{\mathrm{C}}[11]$ to 0.61 when performing unidirectional tensile testing after ECAE.

The Norton-Hoff formula, Eq. (2), uses the strain rate and has a parameter $\mathrm{P}_{3}$ which can be related to the strain rate sensitivity. In terms of elasto-visco-plastic modelling, this is the viscoplastic term. The corresponding value of $P_{3}=m=0.033$ is even higher than the value of $m-0.014$ published by May et al. [12]. It must be remarked that the value of this parameter is given as a side effect of the optimization process and was not the primary goal of the modelling. However, it shows how the material after ECAE does show significant strain rate sensitivity. The effect of this (transient) softening and the (high) strain rate sensitivity explains such a large post-uniform elongation.

When deforming an ECAE sample in tension, the homogeneous deformation is small due to the limited work hardening, while the post-uniform elongation is high. When calculating the correct true stress and true strain by use of the real sample profile, it was found that after a quick strain hardening some softening occurs, followed again by strain hardening. This was also found when using the method described by Segal and coworkers.

The advantage of the FE approach is that the material law accuracy can be validated by simple comparisons between the experimental force-displacement curve and the predicted sample profile. However, the use of EP optimization depends strongly on the given initial values of the parameters. This study confirms that an EP law with softening as provided by Segal is a very good assumption to model ECAE behaviour. The optimizer just yields to a different hardening value at the end of the stress-strain curve. However, one can note that both displacement profile and force can be recovered easily with the EVP law without strong influence of the initial parameters set. This fact is in favour of the actual EVP material behaviour. As long as an isotropic law is assumed, 2D FE analyses are sufficient and a quick answer is provided by FE inverse modelling. The use of an anisotropic Hill yield locus or a more complex model requires a 3D mesh and more computer processing time. However, it will allow the recovery of the A and B values of the elliptical sample cross-section. This work is currently in progress.

\section{Acknowledgment}

This study was carried out in the frame of project IAP, P5/08 financed by the Belgian Science Policy (BELSPO). A.M. Habraken as Research Director of the National Fund for Scientific Research, acknowledges this institution.

\section{References:}

[1] V.M. Segal, S. Ferrasse, F. Alford, Mater. Sci. Eng. A442 (2006) 321-326.

[2] S. Poortmans, B. Verlinden, Mater. Sci. Forum 503-504 (2006) 847-852.

[3] Y. Iwahashi, Z. Horita, M. Nemoto, T.G. Langdon, Acta Mater. 46 (1998) 3317-3331.

[4] S. Poortmans, B. Verlinden, Mater. Sci. Forum 467-70 (2004)1319-1324. 
Published in: Scripta Materialia (2007), vol.56, iss.9, pp.749-752

Status: Postprint (Author's version)

[5] T.J. Turner, M.P. Miller, N.R. Barton, Mech. Mater. (34) (2002) 605-625.

[6] B. Diouf, F. El Houdaigui, S. Poortmans, B. Verlinden, A. Habraken, in: C.A. Mota Soares et al. (Eds.), Proceedings of ECCM III, p. 61/cdrom 2439, Lisbon, 2006.

[7] S. Poortmans, F. El Houdaigui, A. Habraken, B. Verlinden, in: Y.T. Zhu et al. (Eds.), Ultrafine Grained Materials IV, Proceedings TMSConference, San Antonio, 2006, pp. 389-394.

[8] A.M. Habraken, S. Cescotto, Int. J. Numer. Meth. Eng. 30 (1990) 1503-1525.

[9] D.S. Schnur, N. Zabaras, Int. J. Numer. Meth. Eng. 33 (1992) 2039-2057.

[10] B. Peeters, M. Seefeldt, C. Teodosiu, S.R. Kalidindi, P. Van Houtte, E. Aernoudt, Acta Mater. 49 (2001) $1607-1619$.

[11] L. Dupuy, E.F. Rauch, Mater. Sci. Eng. A337 (2002) 241-247.

[12] J. May, H.W. Höppel, M. Göken, Scripta Mater. 53 (2005) 189-194. 